\title{
Computer-Aided Translation in Student's Practical Translation Competence $^{1}$
}

\author{
Dong Chunzhi \\ School of Foreign Languages, Jianghan University, Wuhan, 430056, China \\ whclaradong@163.com
}

Keywords: computer-aided translation (CAT); translation teaching; translation competence

\begin{abstract}
. computer-aided translation system is now used in teaching process to achieve student's practical translation skills training through man-machine interaction translation platform, translation memory, and translation instance mode. Meanwhile, the mode of the division of labor in the translation project can also be employed to train student's cooperative capabilities in translation project management.
\end{abstract}

\section{Introduction}

Currently, the construction of translation majors for bachelor's and master's degrees in China is booming, resulting in the increasing significance of translatology. Consequently, there come the flourishing researches on machine translation and translation software. While the translation teaching aimed to cultivate senior translator can not keep up with their pace accordingly with machine translation and translation software instruction. On the other hand, student' practical translation competence is one of the main training objectives. But traditional translation teaching mode is just a teaching mode of "translation theory / skills integration", a basic approach to improve student's translation skills through a combination of translation theory learning and translation skills drills. This teaching model is basically teacher-centered teaching, with student's initiative and creativity suppressed, thus not conducive to a high level development of student' s integrated translation skills and their ability to evaluate the quality of the translation.[1] This model also lacks real context of translation skills, and is difficult to deal with a variety of practical translation tasks. Moreover, quantitative inadequacy of the translation tasks and species of this translation teaching mode, together with the lack of authenticity of the translated materials, could not achieve the goal of training students' practical translation competence. [2] Thus, the traditional model of translation teaching can not meet the demand in Chinese translation market for student's comprehensive application-oriented translation talents. Presently, the computer-aided translation technology has permeated all aspects of the work of the translator. Translation tools that the translators can use are diversified, greatly saving time and reducing burden of translation work, but also ensuring the translation efficiency. Thus, how to train student's practical translation capacity to meet market demand depends largely on the ability of students to use these translation tools to improve translation quality and adaptation of the information age.

\section{Practical translation competence}

Common views on translation capabilities are: bilingualism, encyclopedic knowledge, the knowledge of translation strategies and techniques, and the technical translation capabilities. Among all these, technical translation competence is particularly important. With the rapid development of computer technology and the Internet, a translator should learn to employ indirect experience to improve the speed and quality of translation. For the translation tasks that they are not too familiar with, a translator can make full use of available monolingual or bilingual corpus, comparing their

\footnotetext{
${ }^{1}$ This work is partially supported by the Teaching and Research Project (No. 2010080), Wuhan Educational Bureau, titled Local College English Majors' "Dual System" Training Mod 
translated version with those from the corpus, and deciding a more authentic expression. For the translation of the term, a translator can also attain aid from CNKI online dictionary or National Science and Technology Terminology Committee's official Website (www.term.gov.cn) to check the standardized expression of terms. Common tools such as these are fundamentally important for a translator to master.

The natures of translation projects on the market are usually in great quantities, with tight schedule and high quality requirements. The traditional translation mode in dealing with these vast amounts of translation tasks is inevitably difficult. Modern project management tools and translation technology must be employed to conform to the market needs. Therefore, to adapt to the social progress, the practical translation competence is not only the basic ability to achieve bilingual and bicultural conversion, but also the capability to master commonly used technical translation tools. General translation software technology can be divided into two types: one involving language conversion and the other not involving language translation, the former including word processing, format conversion, voice input, text optical recognition (OCR), electronic encyclopedias, and the latter including all types of electronic dictionaries, online translation software, network bilingual corpus, paralleled corpus retrieval technology. CAT technology is widely use of various techniques of the information age, greatly improving the efficiency and quality of the translation, and promoting the modernization and industrialization of the translation process. While many CAT technology (such as electronic dictionaries, etc.) have begun to spread, thus making the CAT technology an important target in language research and teaching research, as well as a main means of translation practice. CAT technology is widely used from the start to be developed to function as an assistant translator, to accelerate the translation speed and to reduce duplication of translation work. Such technology meets the needs of the times and the market, and, as a result, shows vigorous power in development. Currently, CAT technology has been widely employed by translation service providers and many multinationals, who claim that professional translators need to master these skills. In today's information age, the level of practical translation competence is commonly measured by the mastery of CAT technology.

\section{Computer-aided translation in translation teaching}

Computer-aided translation (CAT). Computer-aided translation or computer-assisted translation (CAT) starts to provide a translator platform (translator workstation) for professional translators. Computer assisted translation is a technology developed rapidly after the translation materials electronized. [3] The so-called technique, in a broad sense, is the use of technology, such as computer software, hardware, networks, and other auxiliary equipment in the translation process, and the technique on pre-translated text analysis and processing, translation memory, and the proofreading software, as well as desktop publication. In a narrow sense, technique refers specifically to the improvement of the translation process and the development of special software and related technologies.

According to previous studies, the currently used computer-aided translation tools can be roughly divided into two categories: one is a kind of general software and hardware that are not specifically developed for the translation process and computer software. These tools include the commonly used word processing software (such as Microsoft word, Dragon System), electronic dictionaries software (such as OCR, Abby FineReader, OmniPage, TextBridge, Kingsoft, Kingsoft translation, electronic encyclopedias) and related hardware (such as an external storage facility, rewritable optical drives, digital cameras, scanners, etc.).[4] The other kind is based on a bilingual corpus and translation memory technology (Translation Memory, TM), such as the translation software (more popular as Trados, Word Fast, Word-Fisher, Masanobu CAT, DjVu, SDLX, etc.) [3] This kind of software transmit to a computer to do things involving pure memories, such as term matching and automatic search tips, memory and reproduction of highly similar sentences, so as to eliminate translators' efforts for repeatedly searching the terms, and to realize semantic conversion and transmission. In recent years, translation tools keep escalating its version to make them easier to operate than before 
and have more resources and capabilities. For an instance, the computer memory of Google's Lingoes translation experts (lingoes) is many times smaller than that of Kingsoft, but it is more powerful in the service of word search, sentences example and full translation version.

CAT in training student's practical translation competence. Most of translation work done by graduates is often not satisfactory in terms of quality and efficiency, even by those graduates from prestigious universities. The problems are often not only caused from their language basic translation skills, but in a large part, caused by the lack of CAT technology. [5] Since one can not be proficient in all areas of the field of translation, one finds that with a few dictionaries, he has difficulties in starting rendering translation materials that are unfamiliar to him, especially in a time when all fields booming with new things and new terms. As a result, translators should use CAT technologies, such as web search engines, term bases, etc. so that they can easily understand the meaning of various terms and find the specific terms in certain context. In addition, the translation memory and other CAT technology can reduce duplicating effort in translation and improve translation speed, thus saving a lot of time and manpower and completing translation tasks with quality. [6] In order to achieve effective target in professional training, colleges and universities must advance with the times, seize the opportunity to make timely adjustments, and actively expand the CAT technology in translation teaching to promote students' practical capabilities.

Curriculum mode. The Core of training students' practical translation competence is teaching mode. The course aims to develop students' computer-aided translation competence and translators' integration of attainment and technology in the new era. Students can use computer-aided translation software to improve translation capabilities. The objective of the course is to teach students basic computer-aided translation theory, basic knowledge of computer-aided translation systems, including translation system, network translation systems, the translation tools and its operation, such as corpus retrieval software, translation corpus, translation memory software, electronic dictionaries and so on. Meanwhile, computer-aided translation mode also involves vocational training relevant to the expertise of students, text editing capabilities, and the ability to cooperate for a translation task.

CAT process. The advantage of machine-assisted translation software is that the translation team can share the results via maximum convenience of network. When a translator has difficulty in coping with the original text, other translator's translated version can be offered timely as a reference, thus the entire translation team can maintain consistency in terminology. In the process of translation, all clients are connected to a common server, all using the same terminology library resources and corpus resources, and all the translated texts from different translators will be stored on a public server for other translators to use and refer.

Corpus. CAT technology is development according to the construction of corpus. Corpus construction is fundamental for translation memory, which is crucial for the achievement of translation task. Students need to understand the nature of corpus, such as definition, classification, construction, maintenance and so on. The classification of Corpus, particularly parallel corpus, is very important to terms, alignment and translation memories which are frequently used in the later translation process. In this circumstance, students are required to learn to use one or several corpus analysis tools to master the nature and function of several important components, such as key words, word frequency, word list, type / mark ratio, collocation and retrieval, semantic prosody, etc. to make a preliminary analysis of English and Chinese text. The main purpose for students' learning is to find problems and solve problems, such as the limitations of the tools in the process and analyzing Chinese source text, Chinese segmentation problems and solutions, and the difficulties in text correspondence.

Project analysis and termbase. Term consistency can ensure high quality professional translation. For large translation projects, a centralized, electronic, unified terminology database shows great conveniences to online network of a translation team. Students are to learn about the establishment, editing, expansion, management and maintenance of termbase. Students are required to analyze and standardize definitions of some terms since such high frequency terms and expressions will be automatically displayed by the system, thereby enhancing the efficiency in translation. Users can set high-frequency words and byte length according to their own preference. 
Realization of CAT. Computer-aided translation process is the process of using translation memory (i.e., CAT's core technology). Translation memory is a database to store all translated sentences or a paragraph and the corresponding source language sentence or a paragraph segments that are called translation units. The use of database technology of translation memory is to automatically provide translated versions stored previously. The alignment is the respective matches of the original sentence or paragraph segments and the translated versions of the sentence and paragraph segments, the whole process resulting in great significance in the construction of a translation memory. In the translation process, the new translation sentence and paragraph segments and the revised translation versions are added to the database, so the translation memory dynamically increases. What students need to note is that some distortions or incorrect translation may occur when some Chinese-English texts are aligned, thus the process being time consuming.

Instant construction of database. For a large number of translated data (the original text, the electronic translated version), the use of translation memory, is just putting the translated work into databases. In the later translation, as long as the sentence or paragraph has once been translated, computer-aided translation software will promptly sorting out the translated version as translation tips for reference, thus greatly improving the translation efficiency. It can effectively keep the file with original text format. They save a lot of time and energy for translators.

\section{Conclusion}

The translation-memory-based translation software is currently widely used in translation companies. Colleges and universities should be fully aware of the relationship between the CAT teaching and training students' ability to continuously adjust and improve the teaching mode, though these softwares are usually expensive and are high in price in initial investment. And students need to acquire certain computer knowledge and training so that they can easily operate CAT. The development of translation curriculum should be made, considering the experience of CAT teaching mode abroad and the actual situation. The development of translation curriculum should be application-oriented language translation education so as to train the basic market information, complex, high-quality translation skills, thus enhancing students' market competitiveness in the knowledge-based society.

\section{References}

[1] Song Xinke, Zhang Pingli and Wang Detian "Curriculum Design Reform in Application-oriented Translation Talents Training”, Journal of Xi8nxiang University, Vol.24 No. 6., pp.189-191. Dec. 2010.

[2] Li Haijun and Li Gang, “Cultivation of Englishi Major’s Capability,” Chinese University Teaching, no.3, pp. 69-71, 2012.

[3] Qian Duoxiu, "Reflection on the Teaching Mode of Computer-aided Translation Course,” China Translation Journal, No.4, pp.49-754, 2009

[4] Xu Bing,Guo Hongmei and Guo Xiaoli, “Computer-aided Translation Tools in the $21^{\text {st }}$ Century”, Shandong Foreing Languages Teaching Journal, No 4, pp. 79-86, 2007.

[5] Wang Ting, "The Application of translation workshops in Vocational Colleges Teaching Mode,” Journal of Changchun University of Science and Technology, pp 188-189, Vol. 7, No. 2, Feb. 2012.

[6] Zhang Zheng and Yang Yikuan, "Exploration and Application of MTI CAT Practical Ability,” Shanghai Journal of Translators, pp 44-47, No. 2, 2012. 